\title{
BRAF-V600E mutant papillary craniopharyngioma dramatically responds to combination BRAF and MEK inhibitors
}

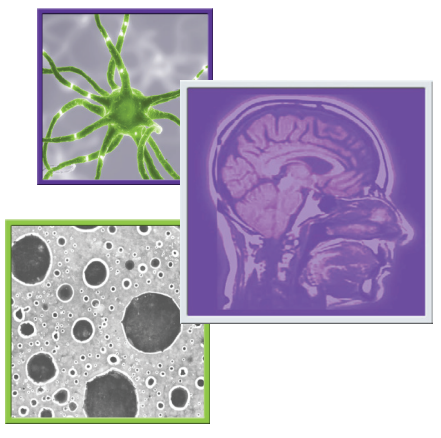

Ashley Roque ${ }^{1,2} \&$ Yazmin Odia* ${ }^{* 1,2}$

\section{Practice points}

- Papillary craniopharyngiomas harbor the BRAF-V600E mutation, adamatinomatous craniopharyngiomas harbor mutations in exon 3 of CTNNB1 ( $\beta$-catenin), and each depicts exclusive epigenetic and expression profiles, highlighting their distinct pathogenesis.

- In addition to melanomas, other solid and hematologic neoplasms harboring BRAF mutations show great response to BRAF with or without concurrent MEK-targeted inhibitors.

- Aside from surgery and radiation, there are no proven therapies for recurrent or residual craniopharyngiomas, which can result in significant morbidity and mortality.

- Given the rarity of papillary craniopharyngiomas, clinical trials of BRAF-directed therapy in craniopharyngiomas prove difficult to study in depth.

- Our case again highlights the oncogenic role of BRAF mutations in papillary craniopharyngiomas and the potential benefit of BRAF-targeted therapy in tumors harboring the BRAF-V600E mutation.

We present a patient with BRAF-V600E mutant papillary craniopharyngioma successfully treated with combination BRAF (dabrafenib $150 \mathrm{mg}$ twice daily) and MEK (trametinib $2 \mathrm{mg}$ daily) inhibitors after her unresectable tumor proved refractory to radiation. Serial brain MRIs and PET revealed marked tumor reduction with gradual neurological improvement and permanent panhypopituitarism.

First draft submitted: 3 August 2016; Accepted for publication: 12 October 2016; Published online: 20 April 2017

Craniopharyngiomas are epithelial tumors arising in the suprasellar region from remnants of Rathke's pouch. The two main pathological subtypes are the adamatinomatous, seen in both children and adults, and the papillary craniopharyngiomas, mainly seen in adults. Regardless of the subtype, craniopharyngiomas are considered 'benign tumors in a malignant location,' because they often cause significant morbidity despite their benign histology [1]. Permanent damage to the optic nerves, pituitary gland, hypothalamus, ventricular system and brainstem can result from both local tumor invasion and post-treatment effects. Vision loss, headaches, endocrine abnormalities and neurobehavioral changes, such as hyperphagia, apathy and decreased libido, are all potential complications frequently observed in patients with craniopharyngiomas [2].

Optimal treatment in craniopharyngiomas remains undefined. The standard therapy is radical resection, when feasible, followed by external beam radiation therapy for residual or recurrent tumor. These

\section{KEYWORDS}

- BRAF V600E

- craniopharyngioma

- dabrafenib • trametinib 
tumors, however, often adhere to the surrounding neurovascular structures of the suprasellar space, making curative surgery exceedingly difficult and postsurgical morbidity common [3]. There is no approved chemotherapy regimen and, until recently, limited data on the molecular mechanisms of tumor growth. Therapeutic options, thus, for recurrent craniopharyngiomas remain limited.

Brastianos et al. recently shed light on a mechanism of pathogenesis in craniopharyngiomas, specifically the first reported $B R A F$ mutations in papillary craniopharyngiomas [4]. Their work suggests that each craniopharyngioma subtype has distinct oncogenic pathways driven predominantly by mutations in single genes. The majority of adamatinomatous craniopharyngiomas harbor a mutation in exon 3 of CTNNB1 $(\beta$-catenin $)$, which encodes a degradation-targeting motif [5]. Papillary tumors, in contrast, characteristically harbor the $B R A F-\mathrm{V} 600 \mathrm{E}$ mutation, which constitutively activates the MEK/ERK pathway. As described by Hölsken et al., adamantinomatous and papillary craniopharyngiomas also harbor distinct epigenomic as well as mutational and transcriptomic profiles [6]. These mutually exclusive gene mutations, methylation patterns and gene expression profiles suggest defining oncogenic pathways and, thus, represent potential targets for therapy $[4,6]$.

The BRAF-V600E mutation has been identified in several cancer types, particularly melanoma, colorectal cancer, non-small-cell lung cancer, papillary thyroid cancer, diffuse gliomas, cholangiocarcinoma, hairy-cell leukemia, multiple myeloma, Langerhans'-cell histiocytosis and Erdheim-Chester disease [7]. The BRAF-V600E mutation is also evident in other benign neoplasms, such ameloblastoma [8]. BRAF-targeted therapy has been proven effective in the treatment of malignant melanoma and other tumor types [7,9-10]. In addition, dual therapy with a MEK-inhibitor improves survival in melanoma compared with BRAF inhibitor monotherapy (Grob, 2015 []). Recent case reports show significant response of craniopharyngiomas to BRAF inhibitors, either monotherapy (vemurafenib) [11] or in combination with a MEK inhibitor (trametinib + dabrafenib) [12], as reported here.

\section{Case history}

We present a woman with a papillary craniopharyngioma harboring the BRAF-V600E mutation that was successfully treated with combination BRAF and MEK inhibition with dabrafenib and trametinib. She presented at 47 years of age with acute vision loss preceded by 4 months of amenorrhea, cold intolerance and headache. A brain MRI showed a 2.7-cm cystic lesion in the suprasellar space with a rim of nodular enhancement. She underwent a right frontal craniotomy for resection, but resection was limited due to tight adherence to the optic apparatus. The residual mass measured $1.1 \mathrm{~cm}$ with nodular enhancement. Postoperatively, her vision improved, but left homonymous hemianopia remained. Her course was complicated by central adrenal insufficiency requiring prednisone. The pathology showed a WHO Grade I papillary craniopharyngioma with positive immunostain for BRAF-V600E (Figure 1). Next-generation sequencing confirmed the presence of BRAFV600E mutation as well as an FGFR3 splice site (437_445+3del12), along with two variants of unknown significance (BRCA2-G1771D and FGFR4-S551F) [13].

One month after her initial resection, her vision worsened in both eyes. Repeat brain MRI revealed an enlarging cystic $(2.4 \mathrm{~cm})$ causing mass effect on the optic chiasm. She underwent Ommaya catheter placement into the cyst to allow for percutaneous aspiration of cyst fluid. For residual tumor, she underwent involved field radiation comprised of $54 \mathrm{~Gy}$ in 30 fractions ending by 3 months after initial presentation.

At 7 months after diagnosis, she developed progressive headaches, behavioral changes and left hemiparesis. A brain MRI revealed significant growth of the cystic tumor upward into the midbrain and right basal ganglion, causing significant mass effect and cerebral edema throughout the right cerebral hemisphere. Intracyst fluid was sampled from the Ommaya and cerebrospinal fluid via a lumbar puncture. Both samples were negative for signs of infection. To help distinguish between radiation-induced necrosis and active tumor growth, serial brain MRI revealed continued progression of areas of enhancement and surrounding edema evident by fluid-attenuated inversion recovery (FLAIR) images, while 18-Fluoro-deoxyglucose PET (FDG-PET) revealed marked glucose uptake in the areas of abnormal enhancement with decreased metabolic activity in right hemisphere, confirming recurrence of tumor with surrounding edema. The hallucinations and behavioral changes improved on aripiprazole, while headaches resolved and vision improved on dexamethasone. Her course was further complicated 


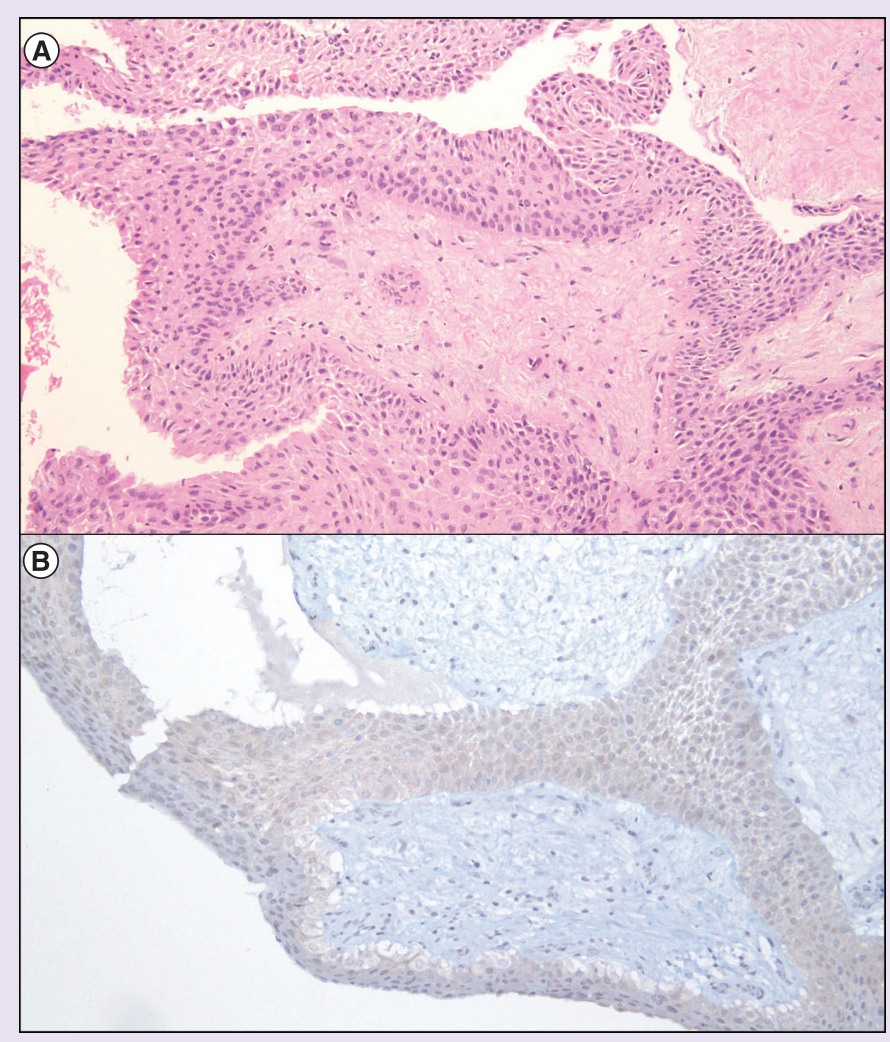

Figure 1. Pathology findings. H\&E-stained section revealed a well-circumscribed neoplasm with well-differentiated squamous epithelium surrounding a hypocellular fibrous stroma with stellate and bipolar-shaped cells (A: 100X). No mitotic figures, atypia or pleomorphism was seen. Immunohistochemistry for mutated BRAF-V600E was positive (B: 100X, Ventana VE1 clone).

H\&E: Hematoxylin and eosin; VE1: BRAF-V600E.

by brittle diabetes insipidous requiring recurrent hospitalizations and maximal medical management.

Given the confirmed BRAF-V600E mutation, she was treated with combination BRAF and MEK inhibitors, specifically dabrafenib 150-mg orally twice daily and trametinib 2-mg orally once daily. A brain MRI after 2 months revealed significant decrease in tumor size, from $2.6 \times 2.3 \times 3.2-\mathrm{cm}$ pretreatment to $2.1 \times$ $1.9 \times 2.3-\mathrm{cm}$ post-treatment. Subsequent brain MRI and FDG-PET at 4 months showed further response to treatment, with decrease in tumor size $(1.6 \times 0.9 \mathrm{~cm}$ at maximal diameter) and reduced volume and intensity of FDG uptake on PET. The FDG-PET also showed restored metabolic activity throughout the right cerebral cortex, consistent with normal cortical activation and corresponding with the improved cognitive and neurologic function. FLAIR MRI reveal near disappearance of the tumor and edema at 7 months after treatment compared with pretreatment. The MRI and PET imaging findings throughout her course are depicted in Figure 2. Concurrent with the radiographic response, her strength, cognition and behavior and her neurological examination gradually and appreciably improved, though her panhypopituitarism proved permanent. Aside from initial intermittent fever, she experienced no significant side effects from her chemotherapy. She continues to improve clinically and radiographically after 7 months of therapy.

\section{Summary}

Optimal treatment of craniopharyngiomas is limited for recurrence after maximal resection and radiotherapy. Craniopharyngiomas and/ or attempted surgical resection often result in notable morbidity. There is no approved chemotherapy for recurrent tumors. Recent 
studies, however, show most adult-onset papillary craniopharyngiomas harbor $B R A F-\mathrm{V} 600 \mathrm{E}$ mutation, which promotes cell growth by constitutively activating the MEK/ERK pathway. Patients with malignant melanoma and hairy cell leukemia harboring the same mutation are successfully treated with BRAF inhibitors with or without concurrent MEK inhibitor. New trials are currently underway to determine the utility of BRAF and MEK inhibitors in other solid tumors. In the literature to date, two patients with a papillary craniopharyngioma showed successful response to BRAF-targeted therapy, either vemurafenib monotherapy [11] or combination BRAF (trametinib) and MEK (dabrafenib) inhibitor therapy [12]. Our case represents the third papillary craniopharyngioma successfully treated with BRAF-targeted therapy. The combination dabrafenib 150-mg orally twice daily and trametinib 2 -mg orally once daily proved well tolerated, aside from the expected and transient fevers. Her tumor had rapid progression after biopsy and involved field radiation, yet combination BRAF and MEK inhibition reduced the tumor by more than $75 \%$ by 5 months, with corresponding marked decrease in metabolic activity in the tumor and normalization of cortical metabolic activity on FDG-PET. While her panhypopituitarism proved permanent, she continues to
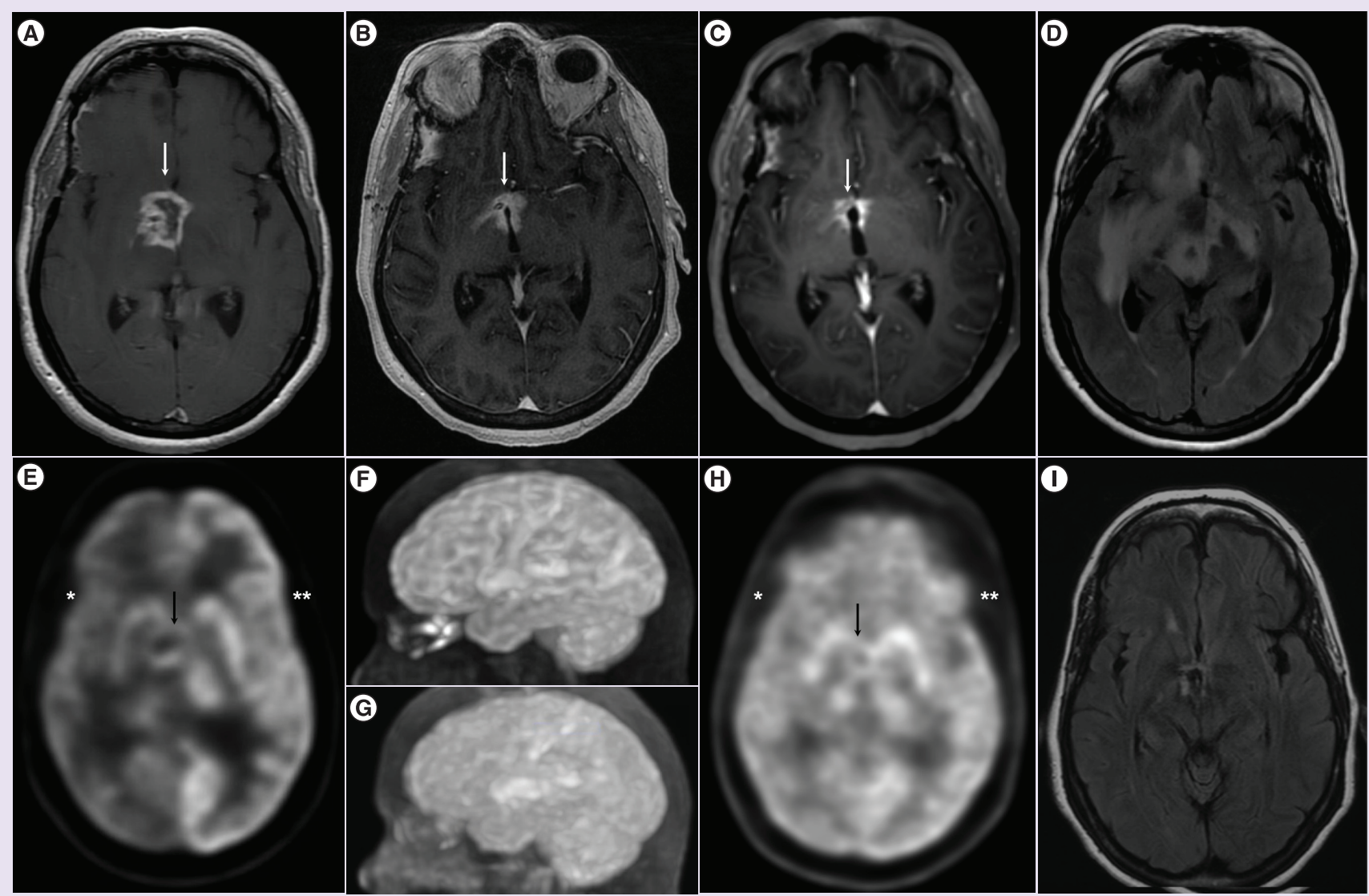

Figure 2. Imaging findings. Axial postgadolinium T1-weighted images on MRI revealed a heterogeneously enhancing mass lesion in the diencephalon (white arrow), anterior and right of the third ventricle (A). The mass continually decreased in size by 2 months (B) and 5 months (C) after initiation of combination BRAF and MEK inhibitors. 18-Fluoro-deoxyglucose PET (FDG-PET) images revealed FDGuptake in a circular mass lesion (black arrow) greatly reduced in size after treatment (G \& H) compared with baseline (E \& F). Cortical metabolic activity in the right hemisphere $(*)$ was markedly decreased compared with the normal contralateral cortex $\left(^{* *}\right)$ in the pretreatment FDG-PET (E), while the bihemispheric FDG uptake normalized post-treatment (H). FLAIR MRI reveal near disappearance of the tumor and edema at 7 months after treatment (I) compared with pretreatment (D).

FDG-PET: 18-Fluoro-deoxyglucose PET; FLAIR: Fluid-attenuated inversion recovery. 
improve neurologically and radiographically after 7 months of therapy.

\section{Conclusion}

This is the third papillary craniopharyngioma harboring the BRAF-V600E mutation to dramatically respond to BRAF-targeted therapy. We anticipate results of ongoing trials of BRAF inhibitors alone or in combination with MEK or AKT inhibitors in BRAF-mutated solid tumors, namely, colorectal carcinoma, ovarian carcinoma and non-small-cell lung carcinoma. Given the rarity of papillary craniopharyngiomas, the efficacy of BRAF-directed therapy for recurrent tumor proves difficult to study in depth. Our case again highlights the oncogenic role of $B R A F$ mutations in papillary craniopharyngiomas. The potential benefit of BRAF-targeted therapy in tumors, such as craniopharyngiomas, harboring the BRAF-V600E mutation is exemplified by this case, but needs confirmation in randomized clinical trials.

\section{Author contributions}

A Roque reviewed the medical record and existing literature and authored the manuscript. Y Odia provided clinical care for the patient, conceptualized the project and critically revised the entire manuscript.

\section{Financial \& competing interests disclosure}

The authors have no relevant affliations or financial involvement with any organization or entity with a financial interest in or financial conflict with the subject matter or materials discussed in the manuscript. This includes employment, consultancies, honoraria, stock ownership or options, expert testimony, grants or patents received or pending, or royalties.

No writing assistance was utilized in the production of this manuscript.

\section{Informed consent disclosure}

The authors state that they have obtained verbal and written informed consent from the patient/patients for the inclusion of their medical and treatment history within this case report.

\section{References}

Papers of special note have been highlighted as:

- of interest; $\bullet$ of considerable interest

1 Gooch MR, Evans JJ, Kenning TJ. Introduction. In: Craniopharyngiomas: Comprehensive Diagnosis, Treatment and Outcome. Evans JJ, Kenning TJ (Eds). Academic Press, Boston, MA, USA, 3-14 (2015).

2 Karavitaki N, Brufani C, Warner J et al. Craniopharyngiomas in children and adults: systematic analysis of 121 cases with long-term follow-up. Clin. Endocrinol. 62(4), 397-409 (2005).

3 Cappabianca P, Cavallo LM, Solari D, Di Somma A, Del Baso De Caro ML. Surgical Approaches - Introduction and outcomes. In: Craniopharyngiomas: Comprehensive Diagnosis, Treatment and Outcome. Evans JJ, Kenning TJ (Eds). Academic Press, Boston, MA, USA, 137-153 (2015).

4 Brastianos P, Taylor-Weiner A, Manley P et al. Exome sequencing identifies $B R A F$ mutations in papillary craniopharyngiomas. Nat. Genet. 46, 161-165 (2014).

- Landmark genomic analysis reveals $B R A F$ mutations as pathogenic in papillary craniopharyngiomas.
5 Sekine S, Shibata T, Kokubu A et al. Craniopharyngiomas of adamantinomatous type harbor beta-catenin gene mutations. $A m$. J. Pathol. 161(6), 1997-2001 (2002).

6 Hölsken A, Sill M, Merkle J et al. Adamantinomatous and papillary craniopharyngiomas are characterized by distinct epigenomic as well as mutational and transcriptomic profiles. Acta Neuropathol. Commun. 4(1), 1-13 (2016).

7 Hyman DM, Puzanov I, Subbiah V et al. Vemurafenib in multiple nonmelanoma cancers with BRAF V600 mutations. N. Engl. J. Med. 373(8), 726-736 (2015).

- Phase II 'basket' study of use of vemurafenib in BRAF V600 mutation-positive non melanoma cancers.

8 Kurppa KJ, Catón J, Morgan PR et al. High frequency of $B R A F \mathrm{~V} 600 \mathrm{E}$ mutations in ameloblastoma. J. Pathol. 232(5), 492-498 (2014).

9 Flaherty KT, Puzanov I, Kim KB et al. Inhibition of mutated, activated $B R A F$ in metastatic melanoma. N. Engl. J. Med. 363(9), 809-819 (2010).

10 Grob JJ, Amonkar MM, Karaszewska B et al. Comparison of dabrafenib and trametinib combination therapy with vemurafenib monotherapy on health-related quality of life in patients with unresectable or metastatic cutaneous $B R A F$ Val600-mutation-positive melanoma (COMBI-v): results of a Phase III, open-label, randomised trial. Lancet Oncol. 16(13), 1389-1398 (2015).

- Landmark trial showing improved efficacy of combined BRAF and MEK inhibition compared with $B R A F$-targeted monotherapy in metastatic melanoma.

11 Aylwin SJ, Bodi I, Beaney R. Pronounced response of papillary craniopharyngioma to treatment with vemurafenib, a BRAF inhibitor. Pituitary 19(5), 544-546 (2016).

-. First report of response to BRAF-targeted therapy for recurrent papillary craniopharyngioma.

12 Brastianos PK, Shankar GM, Gill CM et al. Dramatic response of $B R A F \mathrm{~V} 600 \mathrm{E}$ mutant papillary craniopharyngioma to targeted therapy. J. Natl Cancer Inst. 108(2), pii:djv310 (2016).

-. First report of response to combined BRAF- and MEK-targeted therapy for recurrent papillary craniopharyngioma.

13 FoundationOne Target Panel. http://foundationone.com/docs/Foundation 